\title{
Surface Morphology and Deformation Mechanism of Single Crystal Copper Treated by Laser Shock Peening
}

\author{
Yuanxun Liu ${ }^{1, a}$, Xi Wang ${ }^{2, b}$, Xianqian Wu ${ }^{3, c}$, Chenguang Huang ${ }^{3, d}$ \\ ${ }^{1}$ Institute of System engineering, China Academy of Engineering Physics, Mianyang 621900, China \\ ${ }^{2}$ School of Mechanical, Electronic and Control Engineering, Beijing Jiaotong University, Beijing \\ 100044, China
}

${ }^{3}$ Key Laboratory for Mechanics in Fluid-Solid Coupling Systems, Institute of Mechanics, Chinese Academy of Sciences, Beijing 100190, China

alyx6782006@126.com, ㅎwangxi@bjtu.edu.cn, cwuxianqian@imech.ac.cn, dhuangcg@imech.ac.cn

Keywords: laser shock peening; surface morphology; single crystal; deformation mechanism; microstructure

\begin{abstract}
To study the relation between surface morphology and deformation mechanism of the target material under the shock, a flexible boundary loading, in laser shock peening(LSP), the macroscopic and microscopic surface morphology of a single crystal copper treated by LSP was investigated. The optical profilometer shows a $200-\mu$ m-deep pit forms on the shocked surface under LSP. The optical microscopy shows a set of parallel slip bands appear at the center of the shocked region and many vertical cross slip bands appear at the edge of shocked region. This indicates a large plastic deformation occurs by means of slip for the single crystal copper under LSP and the distributing features of slip bands correspond to the spatial distribution of the shock pressure. The results confirm that the surface morphology of materials under LSP can reflect the deformation mechanism and it can be a new method of studying the deformation mechanism of materials under LSP.
\end{abstract}

\section{Introduction}

LSP is a novel surface treatment technique, which can significantly improve the fatigue life of metals by the effects of laser inducing shock waves ${ }^{[1-4]}$. In LSP process, the laser intensity is on the order of several $\mathrm{GW} / \mathrm{cm}^{2}$ and the pulse time of the laser is on the order of $10 \mathrm{~ns}$. The pulsed high-intensity laser passes through a transparent confined overlay and irradiates the absorption layer on the surface of the target. Then the absorption layer ablates and vaporizes into a plasma. Owing to the expanding of the plasma between the confined overlay and the target, a shock wave with a high amplitude (on the order of several GPa) is generated and propagates into the target. If the amplitude of the shock wave exceeds the Hugoniot elastic limit (HEL) of the target material, plastic deformation occurs. After LSP, residual compressive stresses are induced near the surface of the target and the microstructure is modified, which results to enhance the material performance. Compared with traditional surface treatment like shot peening or cold extrusion for improving the fatigue life, LSP has many advantages: non-contact, no heat-affected zone, more precise control and much better strengthening effects. Thus, LSP has a widely application and has been successfully used in aviation, nuclear and petrochemical industry.

As LSP is a high amplitude and high strain rate $\left(>10^{6} \mathrm{~s}^{-1}\right)$ loading process, the deformation behavior and mechanism of the material treated by LSP is particularly important. The microstructure and deformation mechanism of metallic materials under LSP is one of the focuses. In most previous studies, either the surface or section of the post-peened target is corroded and then observed by optical microscope, or it is observed by transmission electron microscopy ${ }^{[5-7]}$. These ways are a little complex. Besides, little attention are paid to the microstructure of original surface morphology of the peened target and its relation with the deformation mechanism. However, the target bears a flexible boundary loading in LSP, that is to say, the boundary of the loading is a force. Therefore, some characteristics will appear on the shocked surface, which can reflect deformation 
mechanism of the material. In this paper, the single crystal copper, a simple and representative material, was treated by LSP; macroscopic and microscopic surface morphology of the shocked copper was observed and its relation with deformation mechanism was analyzed.

\section{Experimental techniques}

Fig. 1 is the schematic diagram of LSP experiment. The target material is single crystal copper in [100] orientation. The target specimen $(\Phi 10.0 \times 0.8 \mathrm{~mm})$ was cut from the ingot rod using wire-electrode cutting machine. The upper and lower surface of the specimen correspond to the (100) faces. The (100) surface was polished mechanically using grit 200-1200 sandpapers to remove the heat-affected layer and ensure smooth. For the specimen prepared for observing microscopic surface morphology, the (100) face was polished with grit sandpapers and then polished with suspension of $\mathrm{Al}_{2} \mathrm{O}_{3}$. The thickness of the prepared specimen is about $0.8 \mathrm{~mm}$. On the target surface, attached a $0.05-\mathrm{mm}$-thick $\mathrm{Al}$ foil as absorption layer and then confined it by a 4-mm-thick BK-7 glass. The target assembly was fully-clamped with a holder. The LSP experiment was performed with a Q-switched high power Nd: YAG pulse laser having a wavelength of 1064 $\mathrm{nm}$. The output energy of the laser is $2.4 \mathrm{~J}$ per shot. The temporal profile of the laser pulse is in the near Gaussian shape and the full width at half maximum (FWHM) is approximately 10 ns. Its spatial profile is in the nearly Gaussian shape. Different laser intensity can be obtained by adjusting the distance of the target from the laser focusing lens. In this experiment, the radius of laser spot was $1.6 \mathrm{~mm}$ and the laser intensity at the center was about $3.6 \mathrm{GW} / \mathrm{cm}^{2}$. The laser beam was in [100] orientation and irradiated the absorption layer put on the (100) face of the target specimen.

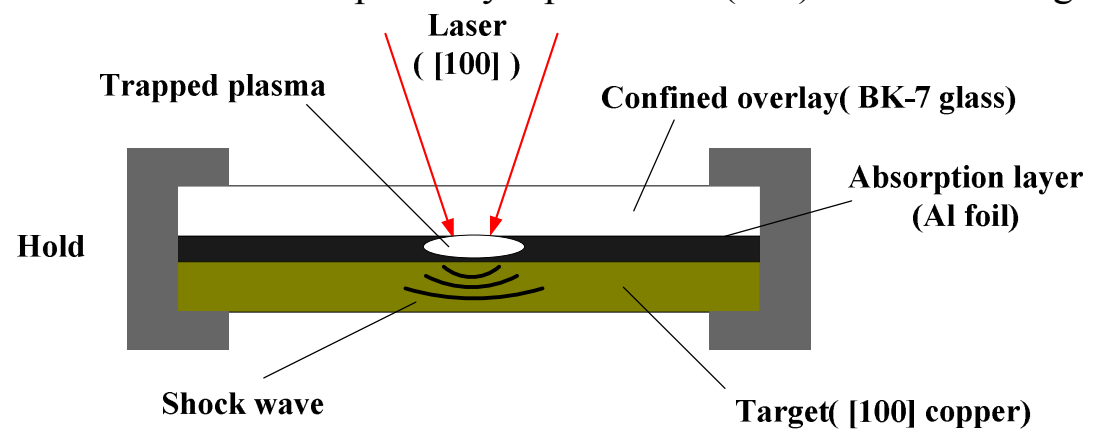

Fig. 1 Schematic diagram of LSP

After LSP, the shocked single crystal copper specimen was washed with acetone, dried and then measured directly. The geometry of the shocked region of the specimen was examinated by means of optical profilometer (LEXT OLS4000 3D) to determine the deformation profile on the macroscopy. And the microscopic surface morphology of the shocked specimen was observed using optical microscopy.

\section{Experimental results and discussion}

Macroscopic surface morphology. As shown in Fig. 2, the shocked single crystal copper deforms significantly and almost totally. The maximum deformation is at the center of the shocked region, about $200 \mu \mathrm{m}$, which is in the same order of the thickness of the specimen $(800 \mu \mathrm{m})$. This reflects that the yield strength of single crystal copper is very low, and under the extremely high shock pressure in LSP, severe plastic deformation occurs in copper. According to the analytical model, which is based on the conservation of energy and proposed by Fabbro et $\mathrm{al}^{[3]}$, the peak of laser-induced shock pressure can be estimated by

$$
p=0.01\left(\frac{\alpha}{2 \alpha+3}\right)^{0.5} Z^{0.5} I^{0.5} \text {. }
$$


In the equation above, $p$ is the peak shock pressure, $\alpha$ is the fraction of the internal energy devoted to the thermal energy (typically, $\alpha=0.25^{[8]}$ ), $I$ is the laser intensity, and $Z$ is the reduced shock impedance between the target and the confined overlay. Here, the shocked absorption layer (Al foil) retained a certain thickness and a transmission coefficient exists between the absorption layer and the target(single crystal copper) while shock waves propagate. Considering the fact, we evaluate the peak shock pressure of single crystal copper by calculating the peak shock pressure of Al foil based on the equation above and multiplying it by the transmission coefficient $T(T=1.4)$. The reduced shock impedance $Z$ between BK-7 glass and Al foil is $1.3 \times 10^{6} \mathrm{~g} \cdot \mathrm{cm}^{-2} \cdot \mathrm{s}^{-1}$, and $I=3.6 \mathrm{GW} \cdot \mathrm{cm}^{-2}$. Finally, the calculated peak shock pressure of single crystal copper is $8.1 \mathrm{GPa}$. Meyers et $\mathrm{al}^{[7]}$ estimated the critical stress for plastic flow of single crystal copper with [001] orientation. The estimated flow stress is $435 \mathrm{MPa}$ and the corresponding critical shock pressure is $669 \mathrm{MPa}$. It's obvious that the peak shock pressure in our experiment is much higher than the critical shock pressure for plastic flow. As a result, the single crystal copper generates a severe plastic deformation.

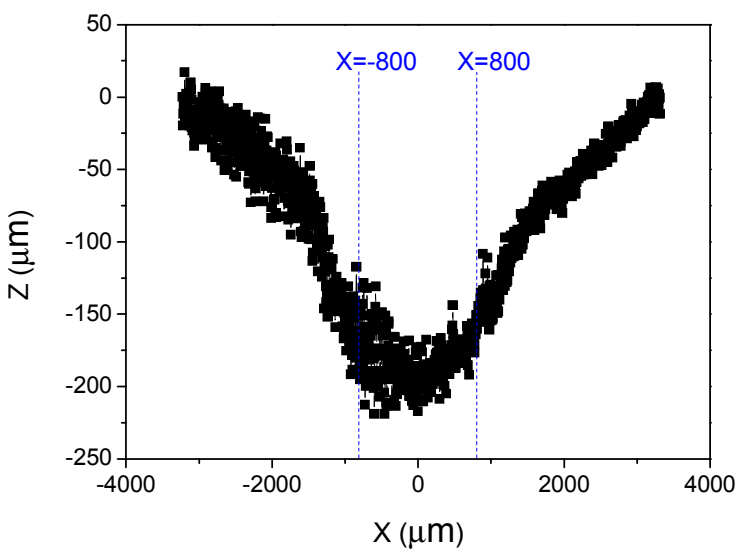

Fig. 2 The typical surface deformation profile of the shocked copper

The deformation is uniform in the central area of shocked region $(-800 \mu \mathrm{m} \sim 800 \mu \mathrm{m})$ while it decreases rapidly at the edge of shocked region. This is due to the spatial distribution of the laser intensity, nearly Gaussian shape; correspondingly, the shock pressure is distributed in Gaussian shape in the shocked region. Thus, the central area suffering plane wave of uniaxial strain and deforms uniformly; the loading reduces quickly at the edge of shocked region, resulting in rapid decrescence of deformation.

Microscopic surface morphology. Fig. 3 shows microscopic surface morphology of single crystal copper after LSP. The deformation microstructure in the shocked suface is mainly slip bands, the space of which is $50-70 \mu \mathrm{m}$. This illuminates that the single crystal copper deforms plastically by slip in the LSP process ( the peak shock pressure is $8.1 \mathrm{GPa}$ ). The result agrees with the experimental result measured by Shcnerder ${ }^{[9]}$ that threshold twinning stress of single crystal copper in [100] orientation is $25 \mathrm{GPa}$, i.e., the copper deforms by slip when pressure is less than 25 GPa.

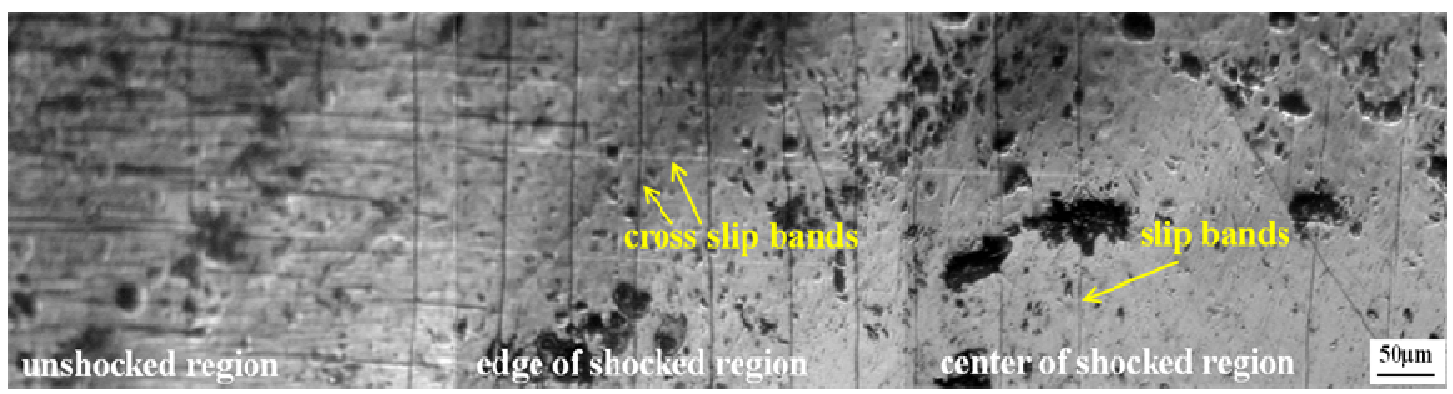

Fig. 3 The microscopic surface morphology of shocked copper 
The characteristics of slip bands in different areas are different (Fig. 3). In the central area of shocked region, a set of parallel slip bands appear. At the edge of shocked region, many vertical cross slip bands appear. This is also due to the distribution of the shock pressure, which is in Gaussian shape in the shocked region. In the central area, the shock pressure is uniform and the material suffers triaxial compression with low shear stress, which results to generate fewer slip bands. At the edge of shocked region, the shock pressure reduces speedily, the material suffers high shear stress, and thus multiple slip occurs, leading to generating a large number of cross slip bands. Finally, severe shear plastic deformation is generated at the edge of the shocked region (Fig. 2). In the face-centered cubic crystal( like copper), slip occurs in $\{111\}$ crystal faces along $<110>$ crystallographic orientation. The intersection lines of slip planes ((111), (-111), (-1-11) and (1-11) crystal faces) and the shocked surface, (100) crystal face, are in [011] and [01-1] orientation. Therefore, the slip bands in [011] and [01-1] orientation generates on the surface of single crystal copper under LSP, and vertical cross slip bands were observed.

\section{Summary}

The macroscopic and microscopic surface morphology of single crystal copper treated by LSP was characterized. The results show a 200- $\mu$ m-deep pit forms on the shocked surface of copper, a set of parallel slip bands appear at the center of the shocked region and many vertical cross slip bands appear at the edge of shocked region. This indicates the shocked copper generated a large plastic deformation by slip and the distributing features of slip bands correspond to the stress condition of copper under the shock pressure distributed in a Gaussian space. This confirms that the surface morphology of materials under LSP can reflect the deformation mechanism and it can be a new method of studying the deformation mechanism.

\section{References}

[1]. C.S. Montross, T. Wei, L. Ye, et al.: International Journal of Fatigue Vol. 24 (2002), p. 1021-1036.

[2]. R. Fabbro, J. Fournier, P. Ballard, et al.: Journal of Applied Physics Vol. 68 (1990), p. 775-784.

[3]. P. Ganesh, R. Sundar, H. Kumar, et al: Materials and Design Vol. 54 (2014), p. 734-741.

[4]. X. Nie, W. He, S. Zang, et al.: Chinese Journal of Lasers Vol. 40 (2013), p. 0803006.

[5]. K.Y Luo, J.Z. Lu, Y.K. Zhang, et al.: Materials Science and Engineering: A Vol. 528 (2011), p. 4783-4788.

[6]. X. Zhu, M. Zhou, Q. Dai, et al.: Journal of manufacturing science and engineering Vol. 131 (2009), p. 054503.

[7]. M.A. Meyers, F. Gregori, B.K. Kad, et al.: Acta Materialia Vol. 51 (2003), p. 1211-1228.

[8]. L. Berthe, R. Fabbro, P. Peyre, L. Tollier and E. Bartnicki: Journal of Applied Physics Vol. 82 (1997), p. 2826-2832.

[9]. M.S. Schneider, B. Kad, D.H. Kalantar, et al.: International Journal of Impact Engineering Vol. 32 (2005), p. 473-507. 


\section{Mechanical and Aerospace Engineering V}

10.4028/www.scientific.net/AMR.1016

\section{Surface Morphology and Deformation Mechanism of Single Crystal Copper Treated by Laser Shock Peening}

10.4028/www.scientific.net/AMR.1016.111

\section{DOI References}

[1] C.S. Montross, T. Wei, L. Ye, et al.: International Journal of Fatigue Vol. 24 (2002), pp.1021-1036. http://dx.doi.org/10.1016/S0142-1123(02)00022-1

[2] R. Fabbro, J. Fournier, P. Ballard, et al.: Journal of Applied Physics Vol. 68 (1990), pp.775-784. http://dx.doi.org/10.1063/1.346783

[3] P. Ganesh, R. Sundar, H. Kumar, et al: Materials and Design Vol. 54 (2014), pp.734-741. http://dx.doi.org/10.1016/j.matdes.2013.08.104

[4] X. Nie, W. He, S. Zang, et al.: Chinese Journal of Lasers Vol. 40 (2013), p.0803006. http://dx.doi.org/10.3788/CJL201340.0803006

[5] K. Y Luo, J.Z. Lu, Y.K. Zhang, et al.: Materials Science and Engineering: A Vol. 528 (2011), pp.47834788.

http://dx.doi.org/10.1016/j.msea.2011.03.041

[6] X. Zhu, M. Zhou, Q. Dai, et al.: Journal of manufacturing science and engineering Vol. 131 (2009), p.054503.

http://dx.doi.org/10.1115/1.4000099

[7] M.A. Meyers, F. Gregori, B.K. Kad, et al.: Acta Materialia Vol. 51 (2003), pp.1211-1228.

http://dx.doi.org/10.1016/S1359-6454(02)00420-2

[8] L. Berthe, R. Fabbro, P. Peyre, L. Tollier and E. Bartnicki: Journal of Applied Physics Vol. 82 (1997), pp.2826-2832.

http://dx.doi.org/10.1063/1.366113

[9] M.S. Schneider, B. Kad, D.H. Kalantar, et al.: International Journal of Impact Engineering Vol. 32 (2005), pp.473-507.

http://dx.doi.org/10.1016/j.ijimpeng.2005.05.010 